\title{
Snow avalanche flow-regime transitions induced by mass and random kinetic energy fluxes
}

\author{
Perry BARTELT, Lorenz MEIER, Othmar BUSER
}

\begin{abstract}
WSL Swiss Federal Institute for Snow and Avalanche Research SLF, Flüelastrasse 11, CH-7250 Davos-Dorf, Switzerland E-mail: bartelt@slf.ch
\end{abstract}

\begin{abstract}
We perform a linear-stability analysis of a system of ordinary differential equations describing the motion of dense flowing avalanches. The depth-averaged equations relate the mean translational velocity of the avalanche to the production of turbulent kinetic energy associated with the random motion of the snow granules. Flow friction is described by an extended Voellmy model, where both the Coulomb and turbulent resistance are related to the random kinetic energy. We identify two snow avalanche flow regimes: (1) an unstable frictional flow regime characterized by low random kinetic energy production and (2) a stable, collisional flow regime at the avalanche front. Flow-regime transitions are governed by the production of random kinetic energy, which is controlled by mass. The unstable regime is characterized by a saddle point which the avalanche encounters at the onset of motion and deposition. Depending on the release mass and/or mass growth, the avalanche head will either fluidize, leading to far-reaching avalanches, or starve and die out. At the tail of the avalanche this saddle point also controls the commencement of deposition and therefore the stopping behaviour of avalanches. We discuss the conditions for flow-regime transitions and the enhanced mobility of snow avalanches.
\end{abstract}

\section{INTRODUCTION}

Recent experimental measurements of the vertical velocity distribution (normal to the flow direction) in full-scale dry and wet snow avalanches obtained from the Swiss Vallée de la Sionne test site (Kern and others, 2009) have been used to develop a constitutive model of flowing snow (Buser and Bartelt, 2009). The idea behind this model is that the internal avalanche velocity is governed by the production of random kinetic energy, which, in turn, is governed by the frictional work rate (Bartelt and others, 2006), itself a function of the avalanche velocity. Higher avalanche velocities, typically found at the avalanche front, are associated with higher random kinetic energy density. The cyclic, nonlinear feedback between the translational velocity (translational kinetic energy, $K$ ) of the avalanche and the random velocity of the snow granules controls the evolution of internal shearing between the front and tail of the avalanche. The constitutive model has been used within the framework of depth-averaged continuum models to predict avalanche run-out, spatial distribution of avalanche deposits, internal and frontal avalanche velocity and impact pressure in general three-dimensional terrain (Christen and others, 2010). The model explains why calibrated Voellmy friction coefficients are required to model extreme avalanche run-out (Buser and Frutiger, 1980; Bartelt and Buser, 2010), a problem of great practical interest (Salm and others, 1990).

In this paper we further examine the role of random kinetic energy, $R$, in snow avalanches. If the production of $R$ is related to the evolution of shear between the front and tail of the slide, it should also be related to observed flow-regime transitions, casually defined as the transition between highvelocity/low-density avalanche fronts and low-velocity/highdensity tails. Indeed, the concepts of shear evolution and flow-regime transitions appear to be complementary in the sense that their mechanical origin and effect are the same, differing only in the terminology of the description, though the concept of a flow-regime transition necessitates a more precise mathematical definition. In the following we define a flow-regime transition as a movement between equilibrium states in the $R-K$ phase plane, derived from a linear-stability analysis of a simplified system of autonomous ordinary differential equations describing avalanche flow. We investigate the stability of the equilibrium points to deduce the solution trajectories in the $R-K$ phase plane and show how these solutions depend, most importantly, on avalanche mass per unit area. Flow-regime transitions appear to exist in all avalanche flow types, both dry and wet. The magnitude of the excursion from equilibrium (the very idea of an avalanche) is controlled by the constitutive parameters characterizing the production and dissipation of random energy.

\section{AVALANCHE MODEL}

The snow avalanche mass per unit area, $M(s, t)$, at time $t$ and location $s$ is given by

$$
M(s, t)=\rho h(s, t) .
$$

The variable $s$ is the arc length along the track profile parameterized by the coordinates $x(s)$ and $y(s), h$ is the mean avalanche flow height and $\rho$ is the density of the avalanche. The avalanche mass can increase or decrease according to

$$
\frac{\mathrm{d} M}{\mathrm{~d} t}=Q(s, t)=Q_{\mathrm{e}}-Q_{\mathrm{d}}
$$

where $Q(s, t)$ represents the mass change rate per unit area. Since we consider the stability of stationary solutions we take $Q(s, t)=0$, indicating the mass influx, $Q_{e}$, is in balance with the mass loss, $Q_{\mathrm{d}}$, or $Q_{\mathrm{e}}=0$ and $Q_{\mathrm{d}}=0$. The equation of motion for the avalanche is

$$
\frac{\mathrm{d}}{\mathrm{d} t}(M u)=M g_{\|}-S,
$$

where $u$ is the mean avalanche velocity in the direction parallel to the slope with angle $\phi(s)$, and $g_{\|}$is the 
gravitational acceleration in the direction of $u, g_{\|}=$ $g \sin \phi(s)$ with $g$ the gravitational constant $\left(9.81 \mathrm{~ms}^{-2}\right)$. Gravitational acceleration normal to the track is denoted $g_{\perp}$, with $g_{\perp}=g \cos \phi(s)$. The angle $\phi(s)$ is defined by the track profile, $\tan \phi(s)=\mathrm{d} y / \mathrm{d} x$. The frictional stress, $S$, is the wellknown Voellmy flow resistance (Voellmy, 1955; Salm, 1993)

$$
S=\mu M g_{\perp}+\frac{M g}{h \xi} u^{2},
$$

where the parameters $\mu$ and $\xi$ represent the dry Coulomb and turbulent friction coefficients derived from the observation of avalanche run-out distances (Buser and Frutiger, 1980; Salm and others, 1990; Bartelt and others, 1999).

Avalanching snow consists of rounded snow granules (Bartelt and McArdell, 2009). The kinetic energy associated with the random movement of the snow granules is defined as $R$ (Bartelt and others, 2006). The total random kinetic energy, $R$, changes along the avalanche track in accordance with the production and decay equation discussed in detail by Buser and Bartelt (2009)

$$
\frac{\mathrm{d} R}{\mathrm{~d} t}=\alpha S u-\beta R .
$$

Parameter $\alpha \in[0,1]$ controls the generation of random energy from the shear work of the mean flow, while parameter $\beta$ (with $\beta \geq 0$ ) accounts for the dissipation of random energy. The value of $\beta$ depends on moisture content and ranges between $0.65 \mathrm{~s}^{-1}$ for dry avalanches and $1.25 \mathrm{~s}^{-1}$ for wet snow avalanches (Buser and Bartelt, 2009; Bartelt and Buser, 2010). The value of $\alpha$ appears to be a function of the properties of the running surface of the avalanche track. At the Vallée de la Sionne site, we find dry and wet snow avalanches have $\alpha=0.10$ (Christen and others, 2010).

The fundamental idea behind this model is to divide the total flow velocity into two separate flows, i.e. the mean flow with kinetic energy $K$

$$
K=\frac{M}{2} u^{2}
$$

and the fluctuating flow with random energy $R$, although both flows are associated with the same mass, $M$. The fluctuation velocities have zero mean, by definition, and can be considered random, especially when far away from the flow boundaries (the bottom and top surface of the avalanche). We disregard the vertical expansion of the flow due to the dispersive pressure associated with $R$, so $R$ effectively does not do mechanical work (Bartelt and others, 2006). At present, we do not allow dispersive pressure (the interaction of $R$ with the basal boundary) to raise the height of the avalanche or to change the flow density. Energy fluxes associated with the production and dissipation of $R$ are therefore irreversible. We account for the influence of random energy on the frictional resistance by formulating Voellmy-Salm parameters $\mu(R)$ and $\xi(R)$ that change with $R$, (Gubler, 1987) according to the relationships:

$$
\mu(R)=\mu_{0} \exp \left(-\frac{R}{R_{0}}\right) \quad \text { and } \quad \xi(R)=\xi_{0} \exp \left(\frac{R}{R_{0}}\right) .
$$

This is a plausible (but heuristic) function to test if we can simulate the changes of $\mu$ and $\xi$ with $R$, where $R$ is given by Equation (5). The fluctuation energy scale, $R_{0}$, that determines the change of the frictional coefficients as a function of $R$ has values in the range $5 \leq R_{0} / h \leq 15 \mathrm{~kJ} \mathrm{~m}^{-3}$, according to measurements at Vallée de la Sionne (Bartelt and Buser, 2010; Christen and others, 2010). The friction coefficients, $\mu_{0}$ and $\xi_{0}$, represent the static Coulomb and turbulent friction coefficients, respectively

$$
\mu(R=0)=\mu_{0} \quad \text { and } \quad \xi(R=0)=\xi_{0} .
$$

This provides $\mu$ values in the range of snow-chute experiments (Platzer and others, 2007). Static dry Coulomb values can be deduced from deposition angles at the front of the avalanche, typically $0.40 \leq \mu_{0} \leq 0.55\left(20-30^{\circ}\right)$, and $\xi_{0}$ $\left(500 \leq \xi_{0} \leq 700 \mathrm{~m} \mathrm{~s}^{-2}\right)$ can be determined from avalanche tail velocities. These $\xi_{0}$ values are similar to values derived by Voellmy (1955); they are smaller than values recommended by the Swiss guidelines (Salm and others, 1990; Gruber and Bartelt, 2007).

Equations (1-3) with constitutive relations Equations (4), (5) and (7) completely specify the dynamics of our system, with the random kinetic energy, $R$, playing a central role. The model requires five constitutive parameters, three limiting the kinetic energy, $K,\left(\mu_{0}, \xi_{0}\right.$ and $\left.R_{0}\right)$ and two describing the production $(\alpha)$ and dissipation $(\beta)$ of $R$.

\section{STABILITY POINTS IN THE $R-K$ PHASE PLANE}

We now perform a linear-stability analysis (Boyce and DiPrima, 1977) of the autonomous system of governing differential equations, Equations (3) and (5),

$$
\frac{\mathrm{d}(M u)}{\mathrm{d} t}=F(R, u) \quad \text { and } \quad \frac{\mathrm{d} R}{\mathrm{~d} t}=G(R, u) .
$$

The variables in Equations (3) and (5) are $u$ and $R$. A steady-state $(\bar{R}, \bar{u})$ is reached when all the variables are time independent, $\dot{R}=0$ and $\dot{u}=0$. Beside the trivial solution $(\bar{R}, \bar{u})=(0,0)$, we seek nontrivial, steady-state points.

By first solving Equation (3) with Equation (4) for $\bar{u}$ we find

$$
\bar{u}^{2}=u_{0}^{2}\left(\exp \bar{r} \sin \phi-\mu_{0} \cos \phi\right),
$$

where $u_{0}^{2}=\xi_{0} h$ and $\bar{r}=\bar{R} / R_{0}$. The relation for $\bar{u}^{2}$ can be substituted into Equation (5) to find a dimensionless equation for $\bar{r}$

$$
H(\bar{r}) \equiv \exp \bar{r}-c-d \bar{r}^{2}=0,
$$

with $c=\mu_{0} / \tan \phi$ and

$$
d=\left(\frac{\beta}{\alpha M g_{\|}}\right)^{2}\left(\frac{R_{0}}{u_{0}}\right)^{2} \frac{1}{\sin \phi} .
$$

The function $H(\bar{r})$ is plotted for several values of $c$ and $d$ in Figure 1 . For flat slopes $(c>1$ and $d$ very large) we find that $H(\bar{r})$ has only one root at high $\bar{r}$. This indicates that to sustain a steady flow state at low slope angles requires a high $R$ production. When the avalanche cannot produce the required $R$, it will stop. Where $\mu_{0}=\tan \phi(c=1)$, we have two steady-state cases and the static equilibrium, $\bar{u}=\bar{r}=$ 0 , if $d$ is large enough. For slopes with $\tan \phi$ slightly higher or lower than $\mu_{0}$ we have two nonzero equilibrium points, depending on the value of $d$. These two points are associated with an upper and lower $\bar{r}$ value. We cannot determine the stability of these equilibrium points analytically. However, numerical solutions reveal the lower equilibrium point is a saddle point and the higher-velocity equilibrium point is stable. This result indicates that for the same slope angle, we can have two different flow regimes appearing at the front or tail of the avalanche. Finally, for steeper slope angles, $c<1$, it is possible to have no equilibrium, depending on the value of $d$, and therefore the avalanche can attain no steady state. 


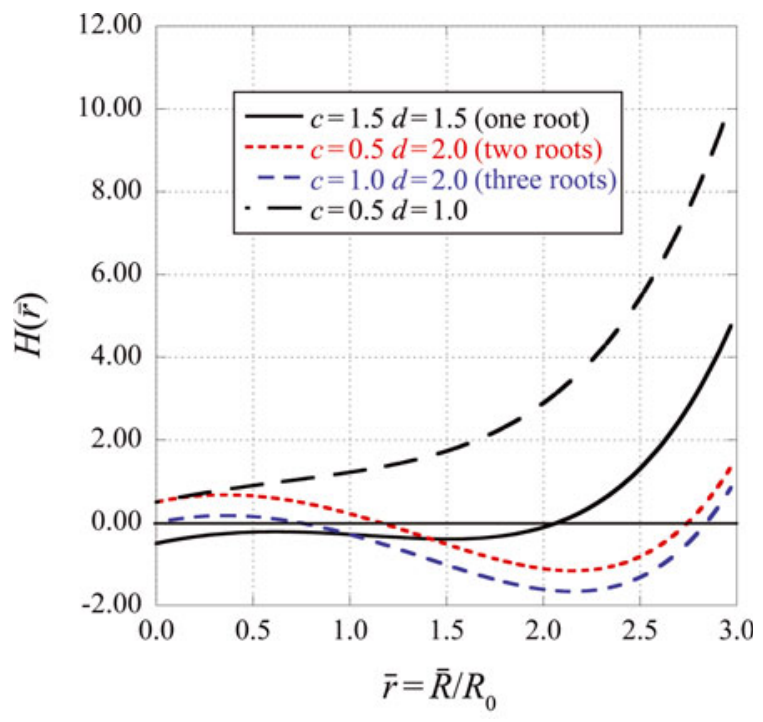

Fig. 1. The function $H(\bar{r})$ for different values of $c$ and $d$. No, one, two or three roots exist, $(\bar{R}, \bar{u})$, depending on the value of $c$ and $d$.

The number of equilibrium points depends on the slope angle and $\mu_{0}$.

To investigate the stability of the system, we construct the Jacobian matrix, J, of the Equation (9) system at the equilibrium (steady-state) point $\bar{R}$ and $\bar{U}$

$$
J=\left(\begin{array}{ll}
\frac{\partial F}{\partial R}(\bar{R}, \bar{u}) & \frac{\partial F}{\partial u}(\bar{R}, \bar{u}) \\
\frac{\partial G}{\partial R}(\bar{R}, \bar{u}) & \frac{\partial G}{\partial u}(\bar{R}, \bar{u})
\end{array}\right) .
$$

The stability of the system Equation $(9)$ at $(\bar{R}, \bar{u})$ is determined by the eigenvalues of $J$. To reach this equilibrium state, the mass, $M$, must be constant and therefore, as stated earlier, $Q(s, t)=0$. Because one cannot solve Equation (11) analytically and because we are interested in a qualitative understanding of our system, we consider the two limiting cases of our friction law separately. The present paper analyzes the two limiting cases $\mu_{0}=0$ and $\xi_{0} \rightarrow \infty$ (described in the two subsections below).

\section{Coulomb friction}

If the $\xi$-dependent friction term is neglected, the total friction, $S$, is given by the Coulomb term, $S=\mu(R)\left(M g_{\perp}\right)$ (case 1 ). For this case, the equilibrium node is

$$
\frac{\bar{R}}{R_{0}}=\ln \left(\mu_{0} \frac{g_{\perp}}{g_{\|}}\right) \quad \text { and } \quad \bar{u}=\frac{\beta R_{0}}{\alpha M g_{||}} \frac{\bar{R}}{R_{0}} .
$$

The eigenvalues, $e$, of $J$ are

$$
e=-\frac{\beta\left(\bar{R}+R_{0}\right)}{2 R_{0}} \pm \frac{\sqrt{\beta^{2}\left(\bar{R}+R_{0}\right)^{2}+4 \alpha g_{\|}^{2} R_{0} M}}{2 R_{0}} .
$$

Since the square root of this expression is always positive, the eigenvalues, $e$, are real and of opposite sign, indicating a saddle point. Solution curves approach $(\bar{R}, \bar{u})$ and then diverge (Fig. 2). At low velocity, $u(0)$, and low $R(0)$ initial conditions, solutions converge to the stopped avalanche case $(\bar{R}, \bar{u})=(0,0)$. For higher initial velocities or random kinetic energies, the avalanche fluidizes, always increasing its velocity and random kinetic energy. This saddle point can be encountered either at the very onset of motion (steep slope) or in the run-out zone (flat slope). The existence of a saddle point indicates that for a particular mass height, $h$,

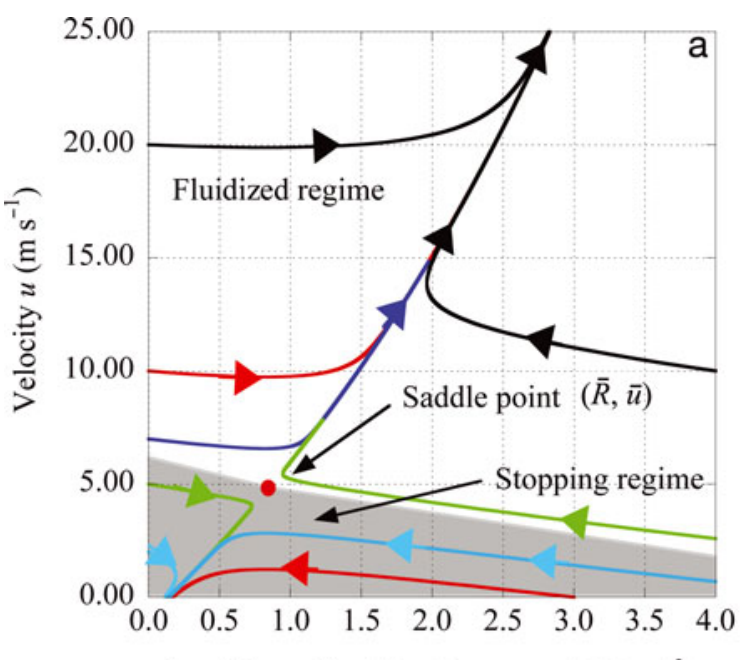

Specific random kinetic energy $R\left(\mathrm{~kJ} \mathrm{~m}^{-2}\right)$

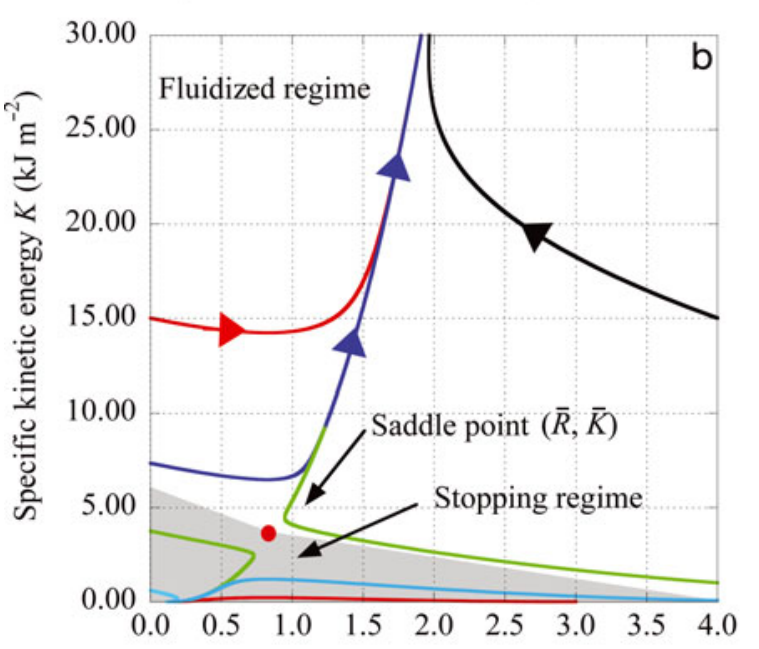

Specific random kinetic energy $R\left(\mathrm{~kJ} \mathrm{~m}^{-2}\right)$

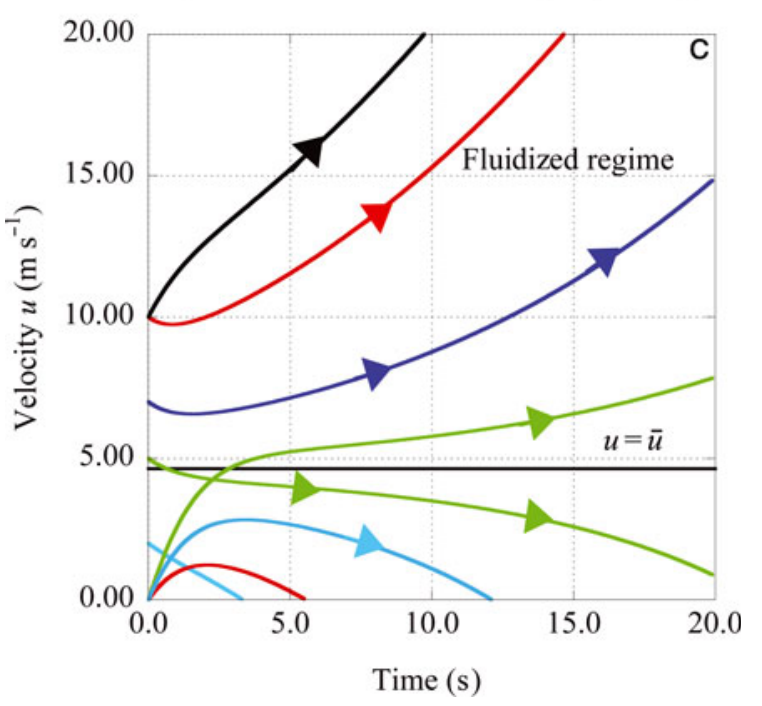

Fig. 2. Numerical solutions of Equations (3) and (5) with differing initial conditions for $u$ and $R$ for the Coulomb friction case. (a) The $R-u$ phase plane. Solution trajectories diverge from an unstable saddle point $(\bar{R}, \bar{u})$. (b) Saddle point in the $R-K$ phase plane. (c) Velocity trajectories. The saddle point separates the stopping or deposition regime from the accelerating, fluidization regime. The stopping regime is encountered at the onset of avalanche motion and controls the beginning of deposition at the avalanche tail. Solution trajectories for a $25^{\circ}$ slope with additional parameters: $\rho=300 \mathrm{~kg} \mathrm{~m}^{-3}, h=1 \mathrm{~m}, \mu_{0}=0.55, \alpha=0.1, \beta=0.8 \mathrm{~s}^{-1}$, $R_{0}=5 \mathrm{~kJ} \mathrm{~m}^{-3}$. 


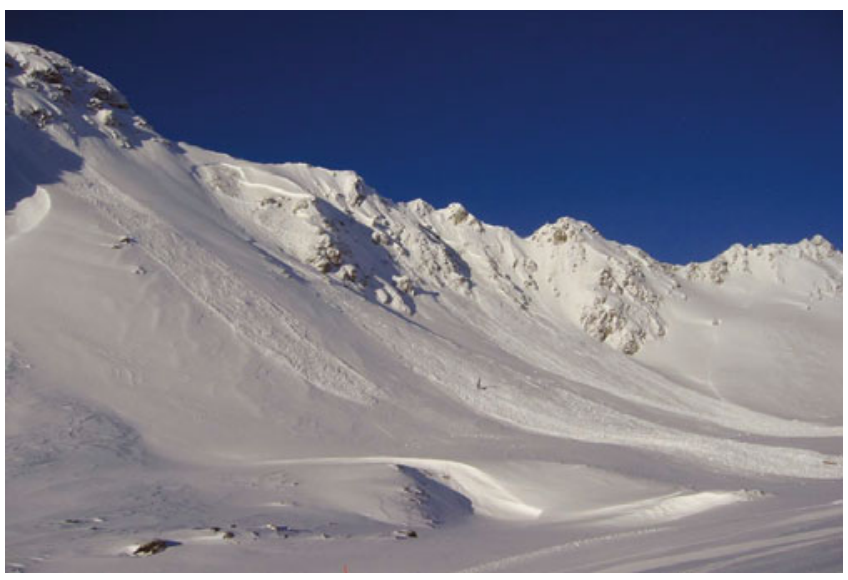

Fig. 3. A series of small avalanches near Davos, Switzerland. A small avalanche has starved on the steep slope. Larger avalanches run out. The mobility of avalanches that begin and stop on slopes with the same slope angle cannot be explained with constant Coulomb friction values.

there exists a slope angle at initiation at which the avalanche will fluidize (fluidized regime; see Equations (14) or (20)), where $\bar{R}$ varies linearly with velocity, mass and the sine of the slope angle. This slope angle varies with avalanche height: lower slope angles are required for fluidization for avalanches with higher flow heights (see Fig. 3). Similarly, in the run-out zone, there exists a slope angle at which avalanche motion will cease (stopping or starving regime; Fig. 2). Cessation of motion can begin at or behind the avalanche front (at the tail), when velocities and random kinetic energy $(R, u)$ decrease to values at which solution trajectories converge to $(0,0)$. Because run-out zone slopes are steeper above the valley bottom, stopping will typically propagate from the slope angle upwards towards the tail, in the form of a stopping front. The relationship between avalanche deposition heights and slope angle reflects the properties of the terrain and the size of avalanche as well as the dissipative properties of the random kinetic energy.

\section{Turbulent friction}

When the avalanche is moving at high speed and turbulent friction is much larger than the Coulomb contribution, $S=$ $(M g) u^{2} / h \xi(R)$ (case 2 ). This high-velocity case is somewhat more difficult to solve since numerical solutions are required to find the equilibrium point, $(\bar{R}, \bar{u})$, and eigenvalues, $e$. However, the system can be approximated by performing a quadratic expansion of the exponential, $\xi(\bar{R})$,

$$
\xi(\bar{R})=\xi_{0} \exp \left(\frac{\bar{R}}{R_{0}}\right) \approx \xi_{0}\left[1+\frac{\bar{R}}{R_{0}}+\frac{1}{2}\left(\frac{\bar{R}}{R_{0}}\right)^{2}+\cdots\right]
$$

and solving for $\bar{u}$ in the resulting quadratic equation,

$$
\left(\frac{a^{2} b}{2}-1\right) \bar{u}^{2}+a b \bar{u}+b=0,
$$

with

$$
a=\frac{\alpha M g_{\|}}{\beta R_{0}} \quad \text { and } \quad b=\xi_{0} h \sin \phi .
$$

The approximate solution for $\bar{u}$ is

$$
\bar{u}=\frac{a b \pm \sqrt{a^{2} b^{2}+4 b\left(1-\frac{a^{2} b}{2}\right)}}{2\left(1-\frac{a^{2} b}{2}\right)},
$$

while the solution for $\bar{R}$ is (cf. Equation (14))

$$
\bar{R}=\frac{\alpha M g_{\|}}{\beta} \bar{u}
$$

We note that real equilibrium steady-state velocities exist only for $\frac{a^{2} b}{2}<2$. Substituting the parameter constants $a$ and $b$ into this relation, we find the following condition for slope angle $\phi$ :

$$
4\left(\frac{\beta R_{0}}{\alpha M g_{\|}}\right)^{2} \frac{1}{\xi_{0} h}>\sin \phi .
$$

One conclusion that we can draw is that on steep slopes no steady solution can be found for large flow heights, $h$ (Fig. 4).

To find the eigenvalues of $/$ we rely on numerical solutions which reveal that the eigenvalues are both real and both negative, indicating an asymptotically stable node (see Fig. 5). Thus, once the avalanche has passed the saddle point given by the Coulomb friction, solution trajectories will be attracted to a high-velocity, high- $R$ equilibrium $(\bar{R}, \bar{u})$. The analysis reveals the importance of the relationship between flow height and slope angle in combination with the ratio $\beta R_{0} / \alpha$, describing the production and decay of random kinetic energy. Steady velocities can be found on flat slopes, especially for large flow heights. When the slope flattens, the flow height can increase and there still exists a steady solution.

\section{DISCUSSION AND CONCLUSION}

The insertion of random kinetic energy fluxes (Equation (5)) into a well-known avalanche dynamics model (Equation (3)) facilitates the mathematical description of flow regimes and flow-regime transitions in snow avalanches. The description allows many salient features of avalanche behaviour to be modelled with simple frictional relationships. We modified the Voellmy (1955) flow law to include the effect of random kinetic energy fluxes. This is in the sense of Voellmy, who remarked in his landmark paper (Voellmy, 1955) that the mobility of flowing snow is similar to vibrated concrete: mobility increases with shaking. Reversible, dilative volume changes, resulting from $R$, produce significant changes in shear strength. This suggests that the constitutive model to describe shear is less important than a mathematical description of how shear strength evolves as a function of the random kinetic energy fluxes.

Our analysis reveals that the regimes are controlled by the production of $R$. The critical equilibrium values of $\bar{R}$ are in both cases directly proportional to the gravitational work rate, $\dot{W}_{\mathrm{g}}$,

$$
\bar{R}=\frac{\alpha}{\beta} \dot{W}_{\mathrm{g}}=\frac{\alpha}{\beta} M g_{\|} \bar{u},
$$

(cf. Equations (14) and (20)) and therefore depend directly on the mass per unit area and the flow height. This helps explain the extreme mobility of avalanches starting with large fracture heights. The larger the flow height, the more likely flow fluidization will occur. If, at the onset of motion of a small slide, the influx of mass is sufficiently large, 


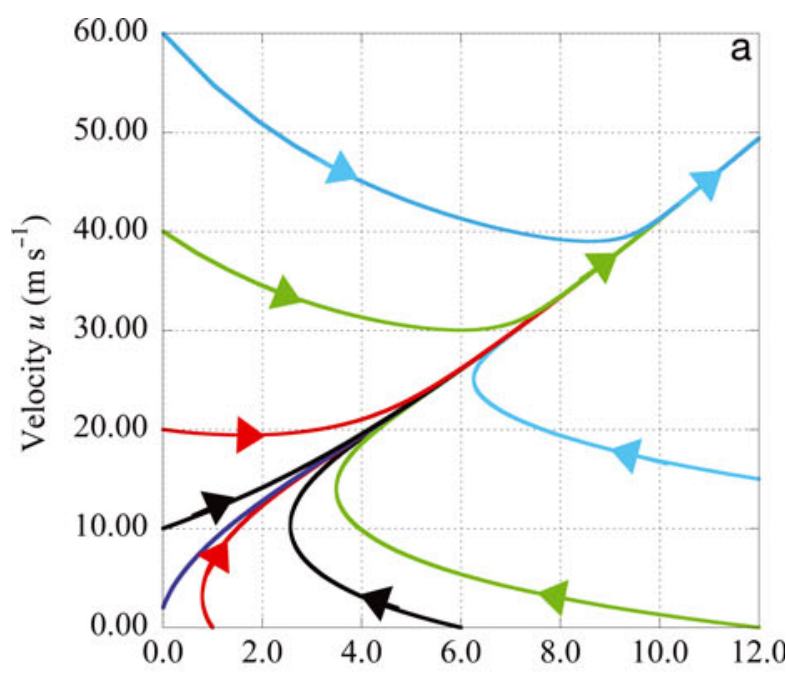

Specific random kinetic energy $R\left(\mathrm{~kJ} \mathrm{~m}^{-2}\right)$

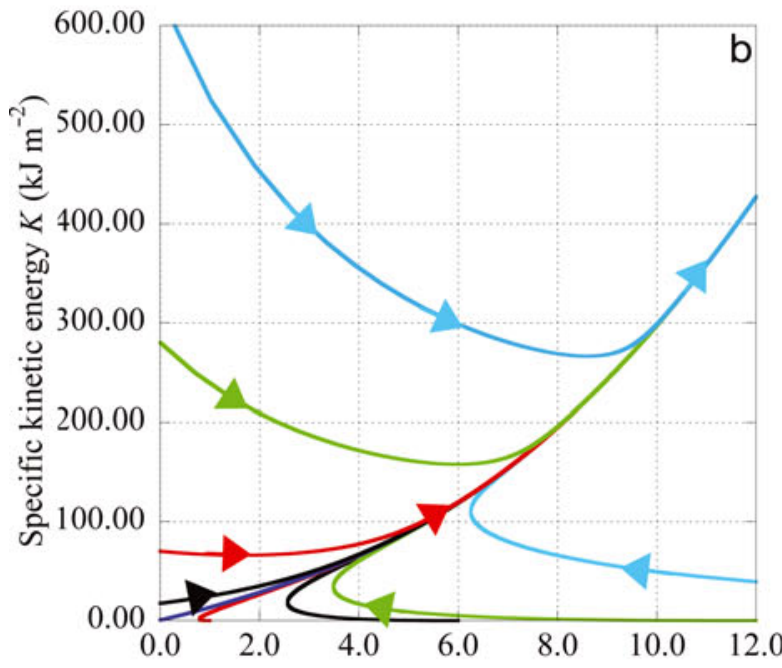

Specific random kinetic energy $R\left(\mathrm{~kJ} \mathrm{~m}^{-2}\right)$

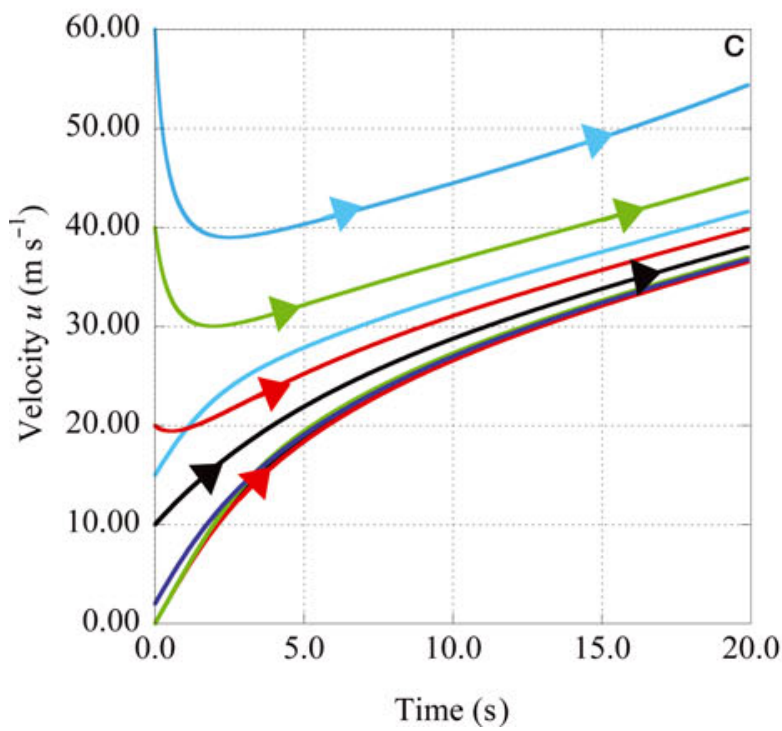

Fig. 4. Numerical solutions of Equations (3) and (5) with differing initial conditions for $u$ and $R$ for the turbulent friction case with no steady solutions. (a) The $R-u$ phase plane. Solution trajectories can diverge $(\bar{R}, \bar{u})$ for large slope angles and large flow heights. (b) $R-K$ phase plane. (c) Divergent velocity trajectories. The solution trajectories for $33^{\circ}$ slope with additional parameters: $\rho=$ $350 \mathrm{~kg} \mathrm{~m}^{-3}, h=1 \mathrm{~m}, \xi_{0}=500 \mathrm{~m} \mathrm{~s}^{-2}, \alpha=0.1, \beta=0.75 \mathrm{~s}^{-1}$, $R_{0}=5 \mathrm{~kJ} \mathrm{~m}^{-3}$
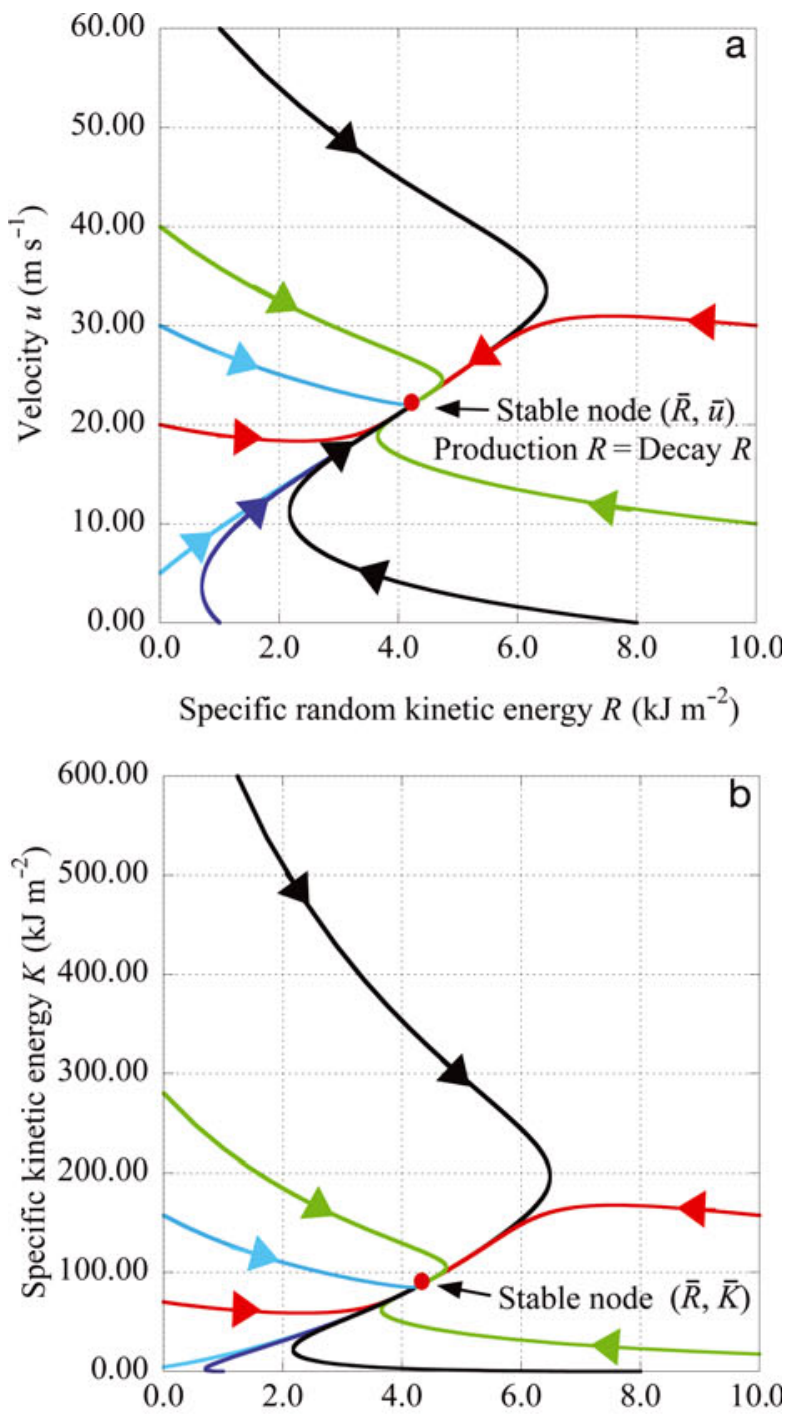

Specific random kinetic energy $R\left(\mathrm{~kJ} \mathrm{~m}^{-2}\right)$

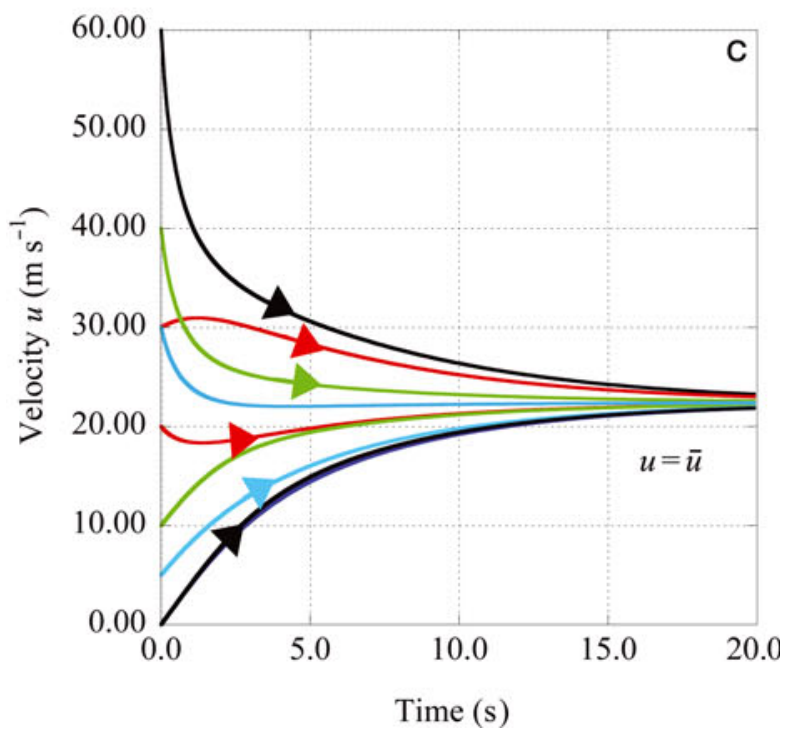

Fig. 5. Numerical solutions of Equations (3) and (5) with differing initial conditions for $u$ and $R$ for the turbulent friction case. (a) The $R-u$ phase plane. Solution trajectories converge to a stable node $(\bar{R}, \bar{u})$. (b) Stable node in the $R-K$ phase plane. (c) Velocity trajectories. The stable node exists only for moderate slope angles. Solution trajectories for a $25^{\circ}$ slope with additional parameters: $\rho=350 \mathrm{~kg} \mathrm{~m}^{-3}, h=1 \mathrm{~m}, \mu_{0}=0.55, \xi_{0}=500 \mathrm{~m} \mathrm{~s}^{-2}, \alpha=0.1$, $\beta=0.75 \mathrm{~s}^{-1}, R_{0}=5 \mathrm{~kJ} \mathrm{~m}^{-3}$. 
the avalanche can also pass the saddle point, defined by Coulomb friction, and fluidize. Therefore, release zone size and fracture height, as well as entrainment thresholds, are necessary to predict avalanche run-out and danger, a fact well known to avalanche mitigation experts. The saddle point also helps explain why small avalanches may stop on steep slopes: at the tail of the avalanche, the gravitational work rate, and therefore the production of random energy, decreases. At some point, solution trajectories will recede to the critical node $(0,0)$. The avalanche will starve as the tail deposits (Fig. 3) (Bartelt and others, 2007). Our analysis indicates that the frictional flow regime of snow avalanches is inherently unstable in the sense that any disturbance at the saddle point will cause the avalanche to fluidize or stop.

The terminal speed of an avalanche varies linearly with $R$; the translational kinetic energy varies with $R^{2}$. This indicates how random energy fluxes, controlled by mass uptake and surface roughness, can control the propagation speed of the avalanche. The collisional flow regime, characterized by turbulent friction, is stable for unchanging boundary conditions. We note that videogrammetric measurements of mixed-powder snow avalanches at the Vallée de la Sionne test site exhibit steady leading-edge velocities (Turnbull and McElwaine, 2007). However, an avalanche might run into conditions where this stable, fluidized regime is lost. This can occur on steep slopes with large flow heights. No steady state is reached and the avalanche will accelerate, reaching high velocities. In that case other dissipation processes must be considered, such as loss of mass (energy) by producing a powder cloud. Such processes are clearly outside the scope of this simple model.

Flow-regime transitions are related to flow density fluctuations. When $R$ does mechanical work at a boundary, density changes, and therefore flow-regime transitions, will result. Flow density cannot decrease without expanding the volume (raising the flow height) of the avalanche, keeping the mass constant. Presently, we do not consider the acceleration that must accompany a rise in the avalanche's centre of mass. We assume $R$ is constant $(\dot{R}=0)$ and therefore the density remains constant. However, the interaction of $R$ with the basal boundary will necessarily result in pushing the bulk of the flow mass upwards. Therefore, density variations are also related to the production of $R$. Higher production rates correspond to lower flow densities. The equilibrium node (attractor) found by considering the turbulent friction, $\xi$, implies an equilibrium flow density at the avalanche front. Thus, the inclusion of random energy fluxes offers the possibility of including density variations, i.e. the movements of the centre of mass in the direction perpendicular to the flow direction. This movement includes an acceleration which in turn produces a reactive, dispersive pressure at the bottom. We suspect that these changes of pressure are within the resolution of our measuring equipment. Our findings will be the subject of a subsequent paper.

\section{ACKNOWLEDGEMENT}

The authors thank D. Issler for his helpful suggestions during the review process. He was able to identify the function $H(\bar{r})$ (Equation (11)) which allowed us to separate the frictional/Coulomb and collisional/turbulent limiting cases with much better physical motivation.

\section{REFERENCES}

Bartelt, P. and O. Buser. 2010. Frictional relaxation in avalanches. Ann. Glaciol., 51(54), 98-104.

Bartelt, P. and B.W. McArdell. 2009. Granulometric investigations of snow avalanches. J. Glaciol., 55(193), 829-833.

Bartelt, P., B. Salm and U. Gruber. 1999. Calculating densesnow avalanche runout using a Voellmy-fluid model with active/passive longitudinal straining. J. Glaciol., 45(150), 242-254.

Bartelt, P., O. Buser and K. Platzer. 2006. Fluctuation-dissipation relations for granular snow avalanches. J. Glaciol., 52(179), 631-643.

Bartelt, P., O. Buser and K. Platzer. 2007. Starving avalanches: frictional mechanisms at the tails of finite-sized mass movements. Geophys. Res. Lett., 34(20), L20407. (10.1029/2007GL031352.)

Boyce, W.E. and R.C. DiPrima. 1977. Elementary differential equations and boundary value problems. New York, John Wiley and Sons.

Buser, O. and P. Bartelt. 2009. Production and decay of random kinetic energy in granular snow avalanches. J. Glaciol., 55(189), $3-12$.

Buser, O. and H. Frutiger. 1980. Observed maximum run-out distance of snow avalanches and the determination of the friction coefficients $m$ and $x$. J. Glaciol., 26(94), 121-130.

Christen, M., J. Kowalski and P. Bartelt. 2010. RAMMS: numerical simulation of dense snow avalanches in three-dimensional terrain. Cold Reg. Sci. Technol., 63(1-2), 1-14.

Gruber, U. and P. Bartelt. 2007. Snow avalanche hazard modelling of large areas using shallow water numerical methods and GIS. Environ. Model. Softw., 22(10), 1472-1481.

Gubler, H. 1987. Measurements and modelling of snow avalanche speeds. IAHS Publ. 162 (Symposium at Davos 1986 - Avalanche Formation, Movement and Effects), 405-420.

Kern, M., P. Bartelt, B. Sovilla and O. Buser. 2009. Measured shear rates in large dry and wet snow avalanches. J. Glaciol., 55(190), 327-338.

Platzer, K., P. Bartelt and M. Kern. 2007. Measurements of dense snow avalanche basal shear to normal stress ratios $(\mathrm{S} / \mathrm{N})$. Geophys. Res. Lett., 34(7), L07501. (10.1029/2006GL028670.)

Salm, B. 1993. Flow, flow transition and runout distances of flowing avalanches. Ann. Glaciol., 18, 221-226.

Salm, B., A. Burkard and H. Gubler. 1990. Berechnung von Fliesslawinen: eine Anleitung für Praktiker mit Beispielen. Eidg. Inst. Schnee- und Lawinenforsch. Mitt. 47.

Turnbull, B. and J.N. McElwaine. 2007. A comparison of powdersnow avalanches at Vallée de la Sionne, Switzerland, with plume theories. J. Glaciol., 53(180), 30-40.

Voellmy, A. 1955. Über die Zerstörungskraft von Lawinen. Schweiz. Bauztg., 73(12/15/17/19), 159-162, 212-217, 246-249, 280-285. 